\title{
A tapered fibre optics biosensor for histamine detection
}

\begin{abstract}
Purpose: This paper aims to estimate the level of histamine in fish and fish products, as it is very important because of their implication in fish poisoning in humans; hence, ascertaining histamine levels in the aforementioned serves as a chemical index for spoilage. Design/methodology/approach: A technique was developed to immobilize an ordered multilayer of diamine oxidase (DAO) by means of chemical cross-linking on the biconical taper surface stepwisely alternating between chitosan, glutaraldehyde and the enzyme. A spectrophotometric signal results from horseradish peroxidase catalyzed reduction of $\mathrm{H} 2 \mathrm{O} 2$, a secondary product of the oxidative deamination of histamine monitored at $450 \mathrm{~nm}$. Findings: The biosensor showed a linear response range up to $1.5 \mathrm{mM}$, a good sensitivity of $0.64 \mathrm{mM}-1$ with detection and quantification limits towards histamine of $0.086 \mathrm{mM}$ (15.8 ppm) and $0.204 \mathrm{mM}(37.7 \mathrm{ppm})$ and a linear response range of $0-1.5 \mathrm{mM}$. It showed a response and recovery time of $14 \mathrm{sec}$ and operational stability up to 40 repeated analyses without significant loss of sensitivity. Practical implications: The developed biosensor has a good potential for use in the quantitative determination of histamine in seafood. Originality/value: The paper described an outcome of an experimental work on tapered fibre optics (taper)-based biosensor coated with DAO embedded into a chitosan membrane to measure histamine.
\end{abstract}

Keyword: Biosensors; Chitosan; Diamine oxidase; Histamine; Tapered fibre optic 